\title{
The effects of sodium oxybate on core body and skin temperature regulation in narcolepsy
}

\author{
ASTRID VAN DER HEIDE ${ }^{1}$, CLAIRE E. H. M. DONJACOUR ${ }^{1,2}$, HANNO PIJL ${ }^{1}$, \\ ROBERTH. A. M. REIJNTJES ${ }^{1}$, SEBASTIAAN OVEREEM ${ }^{3,4}$, GERT \\ J. LAMMERS ${ }^{1,5}$, EUS J.W. VAN SOMEREN ${ }^{6,7}$ and ROLF FRONCZEK \\ ${ }^{1}$ Leiden University Medical Centre, Leiden, the Netherlands; ${ }^{2}$ SleepWake Centre SEIN, Zwolle, the Netherlands; ${ }^{3}$ Sleep Medicine Centre \\ 'Kempenhaeghe', Heeze, the Netherlands; ${ }^{4}$ Radboud University Medical Centre, Nijmegen, the Netherlands; ${ }^{5}$ SleepWake Centre SEIN, \\ Heemstede, the Netherlands; ${ }^{6}$ Department of Sleep \& Cognition, Netherlands Institute for Neuroscience, Amsterdam, the Netherlands; and \\ ${ }^{7}$ Departments of Integrative Neurophysiology and Medical Psychology, Centre for Neurogenomics and Cognitive Research (CNCR), \\ Neuroscience Campus Amsterdam, VU University and Medical Centre, Amsterdam, the Netherlands
}

\section{Keywords}

constant routine protocol, core body temperature, distal-proximal temperature gradient, sleep disorder, sleep onset, slow-wave sleep

\section{Correspondence}

Astrid van der Heide, MD, Department of Neurology and Clinical Neurophysiology, Leiden University Medical Centre, PO Box 9600, NL-2300 RC Leiden, the Netherlands.

Tel.: +31 715262197 ;

fax: +31 715248253 ;

e-mail: a.van_der_heide@lumc.nl

Accepted in revised form 24 March 2015; received 19 January 2015

DOI: $10.1111 /$ jsr.12303

\section{SUMMARY}

Patients suffering from narcolepsy type 1 show altered skin temperatures, resembling the profile that is related to sleep onset in healthy controls. The aim of the present study is to investigate the effects of sodium oxybate, a widely used drug to treat narcolepsy, on the 24-h profiles of temperature and sleep-wakefulness in patients with narcolepsy and controls. Eight hypocretin-deficient male narcolepsy type 1 patients and eight healthy matched controls underwent temperature measurement of core body and proximal and distal skin twice, and the sleep-wake state for $24 \mathrm{~h}$. After the baseline assessment, $2 \times 3 \mathrm{~g}$ of sodium oxybate was administered for 5 nights, immediately followed by the second assessment. At baseline, daytime core body temperature and proximal skin temperature were significantly lower in patients with narcolepsy (core: $36.8 \pm 0.05{ }^{\circ} \mathrm{C}$ versus $37.0 \pm 0.05{ }^{\circ} \mathrm{C}, \quad F=8.31$, $P=0.01$; proximal: $33.4 \pm 0.26{ }^{\circ} \mathrm{C}$ versus $34.3 \pm 0.26{ }^{\circ} \mathrm{C}, F=5.66$, $P=0.03$ ). In patients, sodium oxybate administration increased proximal skin temperature during the day $(F=6.46, P=0.04)$ to a level similar as in controls, but did not affect core body temperature, distal temperature or distal-proximal temperature gradient. Sodium oxybate administration normalised the predictive value of distal skin temperature and distalproximal temperature gradient for the onset of daytime naps $(P<0.01)$. In conclusion, sodium oxybate administration resulted in a partial normalisation of the skin temperature profile, by increasing daytime proximal skin temperature, and by strengthening the known relationship between skin temperature and daytime sleep propensity. These changes seem to be related to the clinical improvement induced by sodium oxybate treatment. A causal relationship is not proven.

\section{INTRODUCTION}

Narcolepsy with cataplexy (narcolepsy type 1) is a sleep disorder characterised by excessive daytime sleepiness, cataplexy, hypnagogic hallucinations, sleep paralysis and impaired maintenance of nocturnal sleep (Dauvilliers et al., 2007). A decreased level of hypocretin-1 (orexin-A) in the cerebrospinal fluid is the hallmark of the disease and is considered to explain all narcolepsy symptoms (Nishino et al., 2000).

Skin and core body temperature play an important role in sleep and wake regulation (Krauchi et al., 1999, 2000; van Someren, 2000). Wake is associated with a relatively low skin temperature and a relatively high core body temperature, 
while sleep is associated with a higher skin temperature and a lower core body temperature. Sleep onset is preceded by a decline in core body temperature and an increase in skin temperature. The decrease in core body temperature is mediated through increased skin perfusion, which consequently leads to the increase in skin temperature, and facilitates cooling of the body (Raymann et al., 2007; van Someren, 2006). These changes are facilitated in part by the postural change from an upright to a supine position that commonly occurs during sleep (Romeijn et al., 2012).

Previous studies demonstrated an altered diurnal profile of skin temperature in narcolepsy. Compared with controls, patients with narcolepsy show an increased distal skin temperature and a decreased proximal skin temperature in the waking state (Fronczek et al., 2006, 2008b; Mayer et al., 1997). This pattern may be considered as characteristic of lowered vigilance (Ramautar et al., 2013; Romeijn and van Someren, 2011) or even 'sleep promoting', as it is also seen in controls immediately before sleep onset. Indeed, temperature manipulation studies in narcoleptic patients counteracting these changes have shown to improve nocturnal sleep and excessive daytime sleepiness (Fronczek et al., 2008a,b). All together, these findings suggest a relationship between hypocretin function, temperature and sleep regulation.

Gamma-hydroxybutyrate (GHB) is a hypnotic used to improve nocturnal sleep and EDS in narcolepsy (Billiard et al., 2006). GHB has a wide range of effects, but the exact mechanisms are still unclear. Altered thermoregulation is one of the effects described in animal studies and human case reports. Rodent studies demonstrate a slight increase in core body temperature after administration of a low dose of GHB (5-10 $\left.\mathrm{mg} \mathrm{kg}^{-1}\right)$ and a clear decrease in core body temperature in higher doses (<500 $\mathrm{mg} \mathrm{kg}^{-1}$; Kaufman et al., 1990). Several studies describe hypothermia in humans with $\mathrm{GHB}$ intoxication (Chin et al., 1998; Krul and Girbes, 2011).

Sodium oxybate (SXB) is the sodium salt of GHB and is registered for the treatment of narcolepsy. Its effects are comparable to the effects of GHB. Given the impact of GHB on temperature regulation, the altered pattern of skin temperature in narcolepsy and the positive effects of SXB on sleep in patients with narcolepsy, it may be hypothesised that the treatment effect of SXB may in part be mediated by its possible restorative effect on temperature regulation. The aim of the present study is to investigate the effect of SXB on core body and skin temperature in relation to its effects on sleep. Therefore, we continuously measured sleep, core body temperature and skin temperature for $24 \mathrm{~h}$ in patients with narcolepsy and controls, before and after 5 days of SXB administration during a constant routine protocol.

\section{MATERIALS AND METHODS}

\section{Subjects}

Eight male patients with narcolepsy (18-65 years old) were included after informed consent. They all fulfilled the criteria for narcolepsy type 1 according to the International Classification of Sleep Disorders-3 (ICDS-3; American Academy of Sleep Medicine, 2014), suffered clear-cut cataplexy and were hypocretin-1 deficient. Two patients were drug-naive, one patient was tapered from antidepressants $\geq 2$ weeks prior to the study, and two patients had prior history with SXB; however, no subject took SXB within 20 days of study initiation. The other patients did not take any medication for at least several months prior to beginning the study. Eight healthy male controls, free of any neurological, endocrine or psychiatric disease, were individually matched for age and body mass index (BMI). Written informed consent was obtained from all subjects. The study was approved by the ethics committee of the Leiden University Medical Centre.

\section{Study design}

The results of this study originate from an extensive, constant routine protocol that was described previously (Donjacour et al., 2011a,b, 2012; Fig. 1).

All subjects stayed overnight in the hospital, and underwent a baseline $24 \mathrm{~h}$ temperature measurement and polysomnography. During this measurement, subjects remained (semi)supine except for bathroom visits. Lights were switched off at 23:00 hours and switched on at 07:30 hours. Subjects were allowed to take daytime naps whenever they wanted. At 08:30, 13:00 and 18:00 hours a standardised cold meal was served, and during the whole day water and tea (caffeine-free) were available. Following the baseline study, subjects ingested SXB for five consecutive days, the 1st and the 5 th day in the hospital. A second $24 \mathrm{~h}$ temperature measurement and polysomnography was performed on the 5 th day of SXB use.

\section{Medication protocol}

To monitor possible side-effects, the first SXB administration was done in the hospital. Because food reduces the bioavailability of the drug, patients were not allowed to eat for at least $2.5 \mathrm{~h}$ prior to drug administration. Subjects received $3 \mathrm{~g} \mathrm{SXB}$ at 23:00 and 03:00 hours. When no adverse-effects were experienced, subjects were allowed to continue the study and used this dosage of SXB for the next 4 nights. The 5th night the subjects spent in the hospital again for the second measurement. Whether subjects were responders or not was not explicitly determined.

\section{Temperature measurement}

During the baseline measurement and 5th night of SXB use, subjects stayed overnight in the hospital and a $24 \mathrm{~h}$ temperature measurement was performed.

Core body temperature was measured with a wireless monitoring system: an ingestible and biocompatible capsule with Vitalsense monitor (Mini Mitter Company, A Respironics, Company, Bend, Oregon, USA; Byrne and Lim, 2007). 


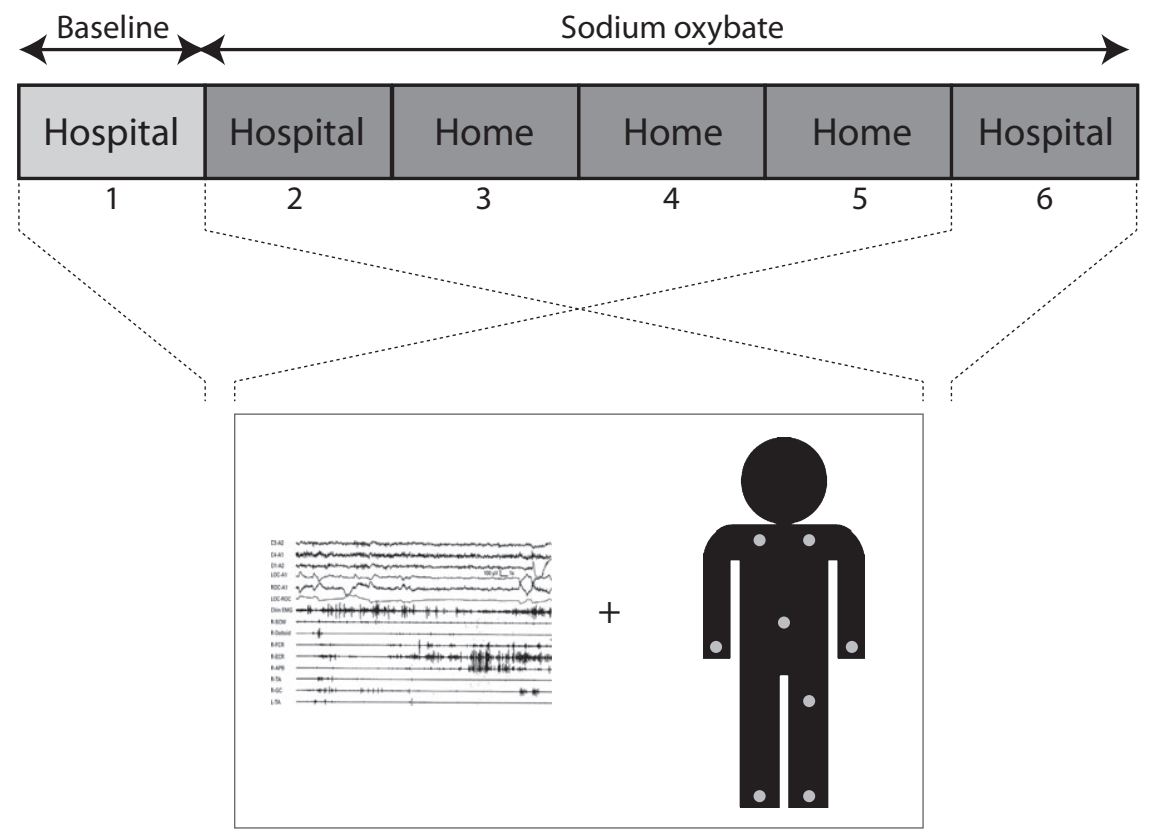

Figure 1. Study design. At day 1, subjects underwent a $24 \mathrm{~h}$ temperature measurement and polysomnography without any treatment. Following this baseline study, subjects received sodium oxybate (SXB) for five consecutive days, the 1st and the 5th day in the hospital. A second $24 \mathrm{~h}$ temperature measurement was performed on the 5th day of SXB use. The dots on the man indicate the location of the iButtons for skin temperature measurement.

Skin temperature was measured wirelessly using Thermochron iButtons (type DS1921H-F50; Maxim Integrated Products, Sunnyvale, CA, USA; van Marken Lichtenbelt et al., 2006). Skin temperature was measured at eight locations: bilateral infra-clavicular area; both hands; abdomen $(1 \mathrm{~cm}$ above the umbilicus); left mid-thigh (musculus rectus femoris); and both feet. Distal skin temperature was obtained from the temperatures at the thenar area at the palmar side of both hands and medial metatarsal area at the plantar sides of both feet (Raymann et al., 2005). Proximal skin temperature contained the infra-clavicular, the thigh and abdominal temperature. Additionally, the distal-proximal temperature gradient [distal minus proximal skin temperature (DPG)] was calculated.

Both core body temperature and skin temperature were sampled once per minute with a temperature resolution of $0.125^{\circ} \mathrm{C}$.

\section{Sleep analysis}

Polysomnographic sleep recordings were performed with a portable, Embletta X100 recorder (Embla, Broomfield, CO, USA) and scored by an experienced sleep technician according to the American Academy of Sleep Medicine criteria (Iber et al., 2007).

Daytime naps were defined as naps if they fulfilled the following criteria: (1) a period of any sleep stage [I, II, III or rapid eye movement (REM)] during the 'lights on' period (between 07:30 and 23:00 hours); (2) for at least two consecutive minutes; (3) all subjects were awake at least 10 min prior to the nap.

\section{Data analysis and statistics}

To compare sleep characteristics between patients and controls, unpaired $t$-tests were used. Paired $t$-tests were used to analyse sleep characteristics before and during SXB administration. Analysis of differences for the number of daytime naps between patients and controls was performed with the MannWhitney $U$-test and the Related-Samples Wilcoxon Signed Rank test because of small group size and skewed distribution.

To evaluate group differences, group by time of day differences and administration by time of day effects on temperature, the mean temperature of each episode of 30 min was calculated. With these data, Generalised Linear Model for repeated-measures with Huynh-Feldt corrections were run using IBM SPSS 20 (SPSS, Inc., Chicago, IL, USA) with between-factor narcolepsy and within-factors SXB and time of day. This analysis was performed on the 24-h data, and separately for daytime (07:30-23:00 hours) and nighttime (23:00-07:00 hours). Post hoc $t$-tests were used to evaluate the times of day where narcolepsy- or SXB-related differences reached significance.

To evaluate the effect of temperature on nap probability in patients at baseline and during SXB administration, mixed effects analysis ( $R$ version 3.1.1, $R$ Foundation for Statistical Computing, Vienna, Austria) was performed. For all analyses, the outcome variable was sleep onset, which was binomially coded for every 30-s epoch as wake $=0$ and sleep onset $=1$ (further sleep epochs were excluded from analysis). As fixed effects, the different temperatures (proximal, distal, core body and DPG), intervention and time (without interaction term) were entered into the model. As random effects, we had 
intercepts for subjects. For each of the temperatures, this analysis was performed with three different regressors. The first regressor evaluated was the temperature during the $30 \mathrm{~s}$ prior to the first sleep epoch. The second and third regressors rather evaluated the predictive value of monotonic changes in temperature prior to sleep onset. To this end, the second regressor was the difference between the temperature immediately prior to the 30-s epoch and the temperature $5 \mathrm{~min}$ before. The third regressor was the difference between the temperature immediately prior to the 30-s epoch and the temperature $15 \mathrm{~min}$ before. $P$-values were obtained by likelihood ratio tests of the full model with the effect in question against the model without the effect in question.

\section{RESULTS}

\section{Subjects}

Eight patients (mean age $38.0 \pm 4.7$ years) and eight controls (mean age $37.9 \pm 4.1$ years) were included after informed consent. The mean BMl was $28.1 \pm 1.6 \mathrm{~kg} \mathrm{~m}^{-2}$ for patients and $27.4 \pm 1.4 \mathrm{~kg} \mathrm{~m}^{-2}$ for controls.

\section{Sleep}

Sleep characteristics are given in Table 1. During the day, patients were significantly less awake compared with controls $(P=0.004)$. SXB administration resulted in significantly less stage $\mathrm{I} / \mathrm{Il}$ sleep during the day $(P=0.049)$, and a trend towards more wake $(P=0.052)$ was seen. SXB intake demonstrated a significantly higher percentage of slow-wave sleep (SWS) during the night in patients $(P=0.014)$ and in controls $(P=0.045)$. SXB administration did not result in change in the prevalence of sleep-onset REM periods either during daytime or during night-time sleep onset.

Daytime napping occurred in all patients at baseline and during SXB administration, and varied from three to 16 naps per patient at baseline, and from three to 11 naps per patient during administration. At baseline, three controls took two or three daytime naps per person, while during SXB administration five controls took one nap. Both at baseline and during SXB administration, patients had significantly more daytime naps than controls (baseline number of naps for patients and controls, respectively: $N=57$ and $N=8, P<0.01$; number of naps during SXB administration for patients and controls, respectively: $N=46$ and $N=5, P<0.01$ ). No significant improvement in the number of daytime naps was seen in controls $(P=0.334)$ or in patients $(P=0.248)$ during SXB administration.

\section{Temperature in patients with narcolepsy versus controls at baseline}

Temperature profiles are shown in Fig. 2, and the results of statistical analysis in Table 2. Patients had a significantly lower core body temperature. Proximal skin temperature showed a trend to be lower in patients $(F=4.13$, df $=1$, $P=0.06$ ), while in distal skin temperature and in DPG no significant differences were found. Analysis of the effect of group by time of day showed a nearly significant effect of narcolepsy by time of day for proximal skin temperature $(F=2.24$, df $=5.49, P=0.05)$.

Post hoc tests indicated a significantly $(P<0.05)$ lower core body temperature in narcolepsy between 16:30 hours and midnight (00:00 hours), and between 10:00 and 12:00 hours the next morning. The same was found in proximal skin temperature between 15:30 and 23:00 hours, and between 11:00 and 12:00 hours the next morning.

Separate analysis of daytime and night-time temperatures demonstrated a significantly lower proximal skin temperature $(F=5.66$, df $=1, P=0.03)$ and core body temperature $(F=$ 8.31, df $=1, P=0.01$ ) in patients during daytime. Furthermore, a significant effect of group by time of day was seen for

\begin{tabular}{|c|c|c|c|c|c|c|c|}
\hline & \multicolumn{3}{|c|}{ Patients $(\mathrm{N}=8)$} & \multicolumn{3}{|c|}{ Controls $(\mathrm{N}=8)$} & \multirow{2}{*}{$\begin{array}{l}\text { Patients versus } \\
\text { controls (baseline } \\
\text { P }\end{array}$} \\
\hline & Baseline & $S X B$ & $\mathrm{P}$ & Baseline & $S X B$ & $\mathrm{P}$ & \\
\hline Wake day (\%) & $80.4 \pm 4.1$ & $84.9 \pm 3.3$ & 0.052 & $96.5 \pm 2.2$ & $98.4 \pm 1.0$ & 0.333 & $0.004 * *$ \\
\hline Wake night (\%) & $25.8 \pm 5.7$ & $19.2 \pm 4.3$ & 0.064 & $18.5 \pm 4.0$ & $19.2 \pm 5.8$ & 0.484 & 0.316 \\
\hline Stage I/II day (\%) & $14.7 \pm 2.9$ & $11.2 \pm 2.6$ & $0.049 *$ & $2.6 \pm 1.7$ & $1.6 \pm 1.0$ & 0.463 & $0.003 * *$ \\
\hline Stage I/II night (\%) & $55.1 \pm 2.5$ & $53.4 \pm 3.7$ & 0.497 & $65.5 \pm 5.7$ & $56.4 \pm 5.2$ & 0.078 & 0.117 \\
\hline SWS day (\%) & $2.1 \pm 0.6$ & $2.7 \pm 1.1$ & 0.526 & $0.05 \pm 0.05$ & $0.05 \pm 0.05$ & 0.356 & $0.013^{*}$ \\
\hline SWS night (\%) & $6.5 \pm 1.9$ & $16.5 \pm 3.0$ & $0.014 *$ & $7.2 \pm 2.0$ & $18.5 \pm 2.4$ & $0.045^{*}$ & 0.818 \\
\hline REM day $(\%)$ & $4.3 \pm 1.7$ & $1.2 \pm 0.5$ & 0.050 & $0.8 \pm 0.5$ & $0.0 \pm 0.0$ & 0.175 & 0.077 \\
\hline REM night (\%) & $12.6 \pm 3.0$ & $10.8 \pm 2.1$ & 0.309 & $8.8 \pm 1.8$ & $5.8 \pm 2.3$ & 0.133 & 0.305 \\
\hline Sleep time day (min) & $254.1 \pm 64.4$ & $140.9 \pm 30.8$ & 0.117 & $32.4 \pm 20.7$ & $15.0 \pm 9.3$ & 0.326 & $0.010 *$ \\
\hline $\begin{array}{l}\text { Sleep time } \\
\text { night (min) }\end{array}$ & $378.4 \pm 29.2$ & $411.9 \pm 22.0$ & 0.064 & $415.6 \pm 19.7$ & $411.9 \pm 29.5$ & 0.484 & 0.316 \\
\hline
\end{tabular}

Percentages of sleep stages during the $24 \mathrm{~h}$ of study, before and during SXB administration. Data are shown as mean \pm SEM. REM, rapid eye movement; SWS, slow-wave sleep; SXB, sodium oxybate. ${ }^{*} \mathrm{P}<0.05$; $* * P<0.01$. 
(a)

Distal skin temperature

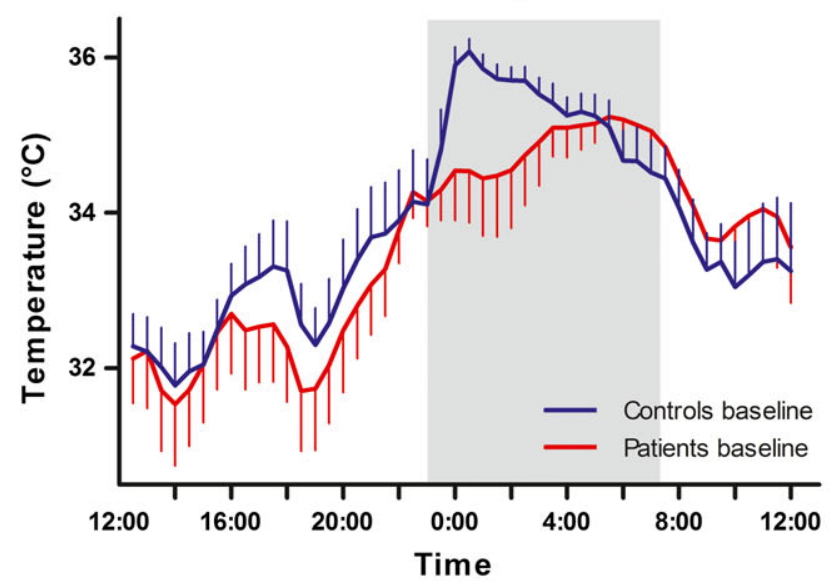

(c) Distal-proximal temperature gradient (DPG)

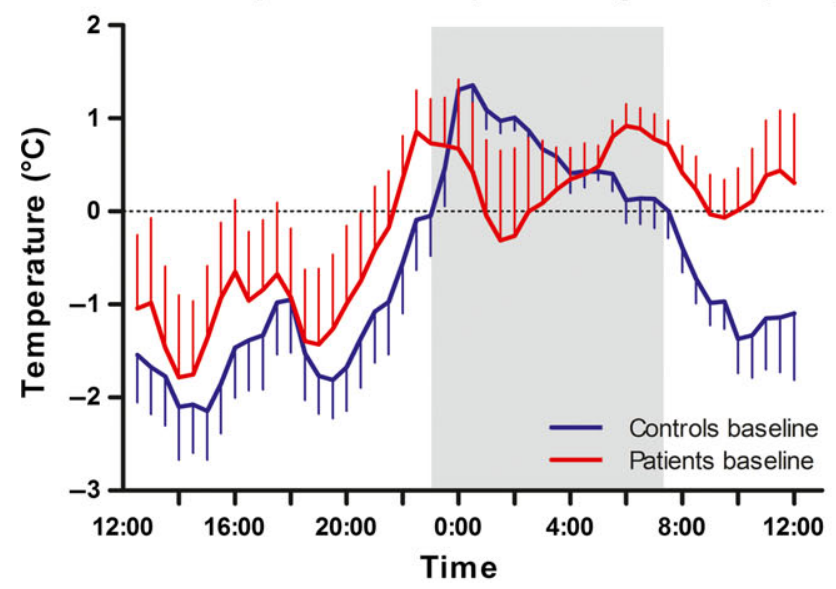

(b)

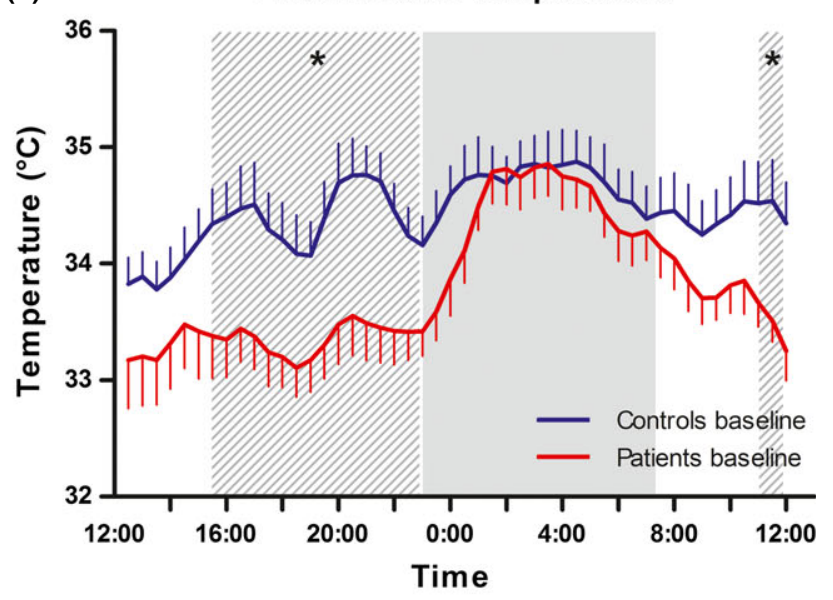

(d) Core body temperature

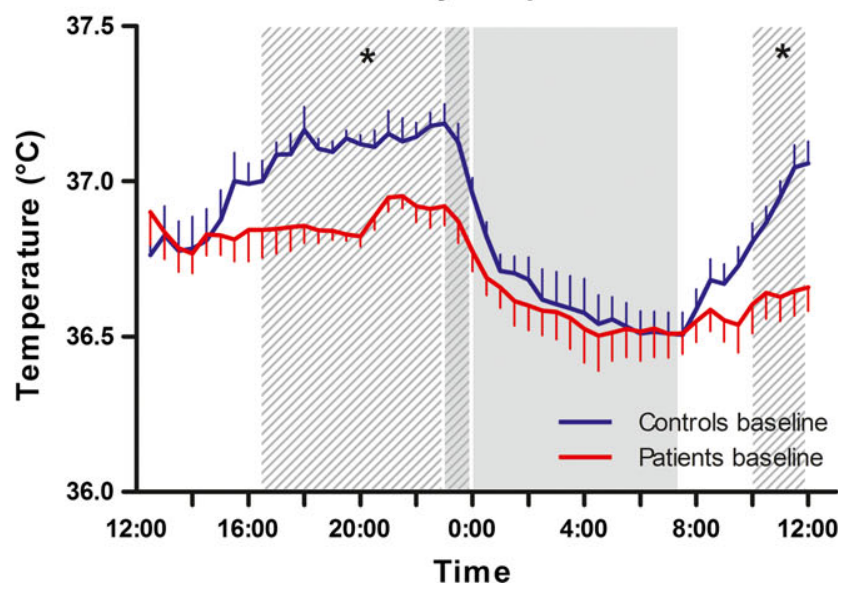

Figure 2. Mean \pm SEM temperature profiles patients versus controls. (a) Distal skin temperature in patients and controls at baseline. (b) Proximal skin temperature in patients and controls at baseline. (c) Distal-proximal temperature gradient (DPG) in patients and controls at baseline. (d) Core body temperature in patients with narcolepsy and controls at baseline. The grey area indicates the lights off period and the striped area the period during which the temperature significantly differed according the post hoc tests $(* P<0.05)$.

Table 2 Results of analysis of temperatures of controls versus patients at baseline

\begin{tabular}{llll}
\hline & $d f$ & $\mathrm{~F}$ & P-value \\
\hline Group effect & & & \\
$\quad$ Proximal skin temperature & 1 & 4.13 & 0.06 \\
$\quad$ Distal skin temperature & 1 & 0.17 & 0.69 \\
DPG & 1 & 0.52 & 0.48 \\
$\quad$ Core body temperature & 1 & 6.46 & $0.02^{*}$ \\
Group by time of day effect & & & \\
$\quad$ Proximal skin temperature & 5.49 & 2.24 & 0.05 \\
$\quad$ Distal skin temperature & 7.46 & 1.25 & 0.28 \\
$\quad$ DPG & 8.91 & 1.75 & 0.09 \\
Core body temperature & 4.83 & 1.96 & 0.10 \\
\hline DPG, distal-proximal temperature gradient. & & \\
*P $<0.05$. & & & \\
\hline
\end{tabular}

core body temperature during daytime $(F=2.82, \mathrm{df}=7.11$, $P=0.01$ ) and for distal skin temperature during night-time $(F=4.34, \mathrm{df}=2, P=0.02)$.

\section{Temperature in patients with narcolepsy: baseline versus SXB}

In patients, a significant main effect of SXB on proximal skin temperature $(F=6.41, \mathrm{df}=1, P=0.04)$ as well as a nearly significant SXB by time of day effect $(F=2.22$, df $=4.80$, $P=0.08$ ) was seen (Fig. 3; Table 3). Additional separate daytime and night-time analyses demonstrated that proximal skin temperature was higher during the day in the SXB condition $(F=6.46$, df $=1, P=0.04)$, but no difference was found during night-time $(F=0.08, \mathrm{df}=1, P=0.79)$. In post hoc tests, significance $(P<0.05)$ was reached from 15:00 to 16:00 hours, from 18:00 to $19: 30$ hours, and from 08:00 to 09:30 hours and 10:30 to 12:00 hours the next morning.

For core body temperature, distal skin temperature and DPG, no main significant effect was found for SXB administration.

Summarising, SXB administration in patients increased proximal skin temperature at several time points during 
(a)

Distal skin temperature

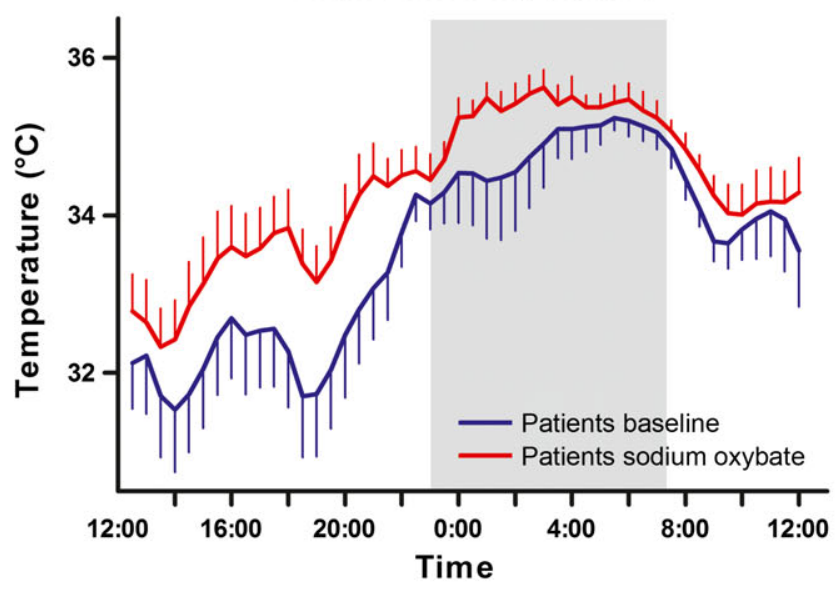

(c)

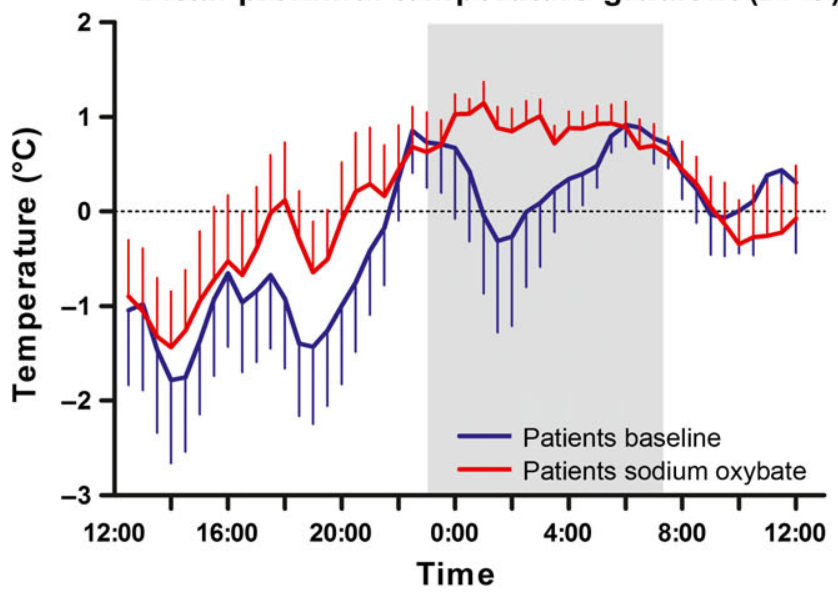

(b)

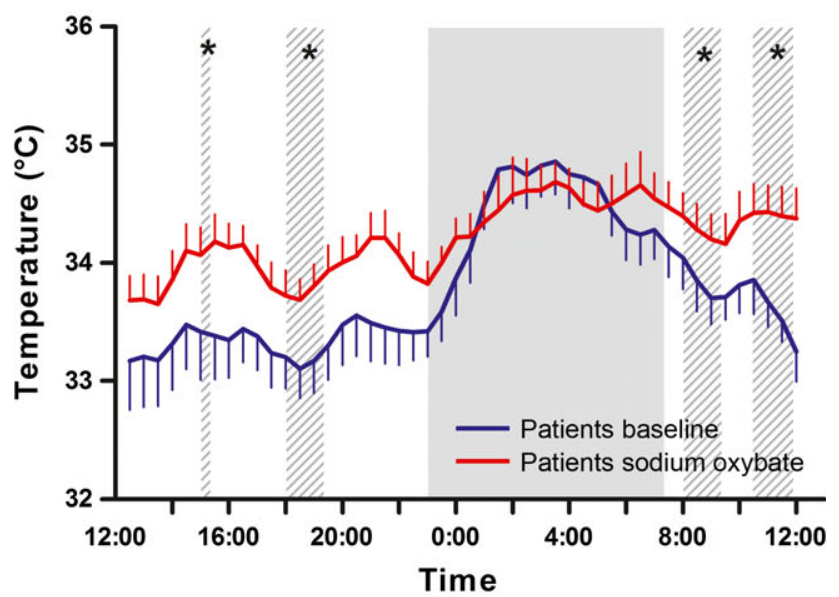

(d)

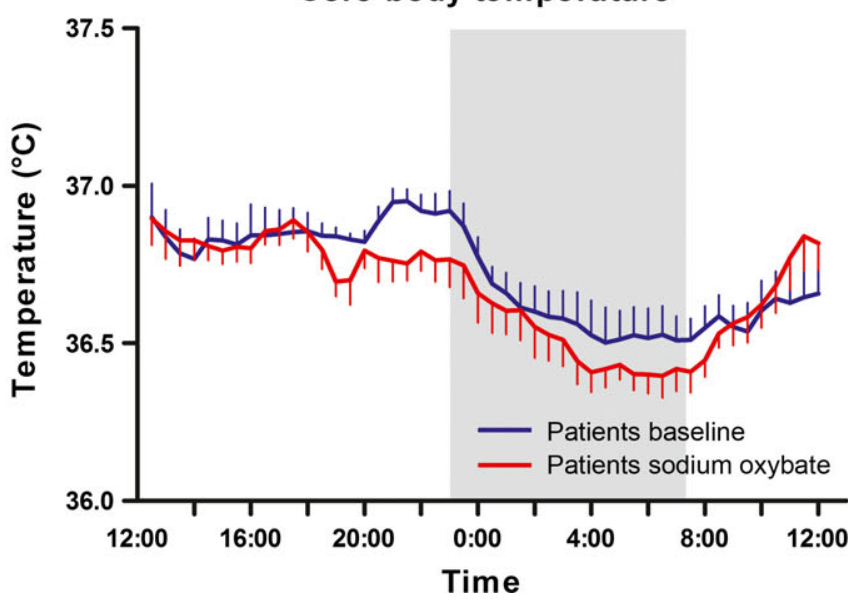

Figure 3. Mean \pm SEM temperature profiles patients at baseline and during sodium oxybate (SXB) administration. (a) Distal skin temperature in patients at baseline and during SXB administration. (b) Proximal skin temperature in patients at baseline and during SXB administration. (c) Distal-proximal temperature gradient (DPG) in patients at baseline and during SXB administration. (d) Core body temperature in patients with narcolepsy at baseline and during SXB administration. The grey area indicates the lights off period and the striped area the period during which the temperature significantly differed according to the post hoc tests $(* P<0.05)$.

daytime. There was no effect on core body temperature, distal skin temperature and DPG.

\section{Temperature in controls: baseline versus SXB}

In controls, no significant effect of SXB or SXB by time of day was found on core body temperature, skin temperatures and DPG (Table 3).

\section{The predictive value of temperature changes on the onset of daytime naps}

Because daytime napping was rare in controls, only daytime naps in patients were analysed (Table 4). Mixed effects analysis of sleep onset in patients at baseline revealed predictive effects of change in proximal skin temperature during the 5 min prior to sleep onset, distal skin temperature and DPG during the 15 min prior to sleep onset. During SXB administration the same effects were seen for distal skin temperature and DPG, supplemented with a predictive value of proximal skin temperature, distal skin temperature and DPG during the epoch prior to falling asleep. No predictive value of core temperature was seen for daytime sleep onset, either at baseline or during SXB administration.

\section{DISCUSSION}

The aim of this study was to investigate the effects of SXB on core body and skin temperature in relation to its effects on sleep in patients suffering from narcolepsy type 1 . This is the first study in which both core body and skin temperature were measured in combination with continuous sleep registration in narcolepsy. At baseline, patients had significantly lower daytime core body and proximal skin temperatures compared 


\begin{tabular}{|c|c|c|c|c|c|c|}
\hline & \multicolumn{3}{|c|}{ Controls } & \multicolumn{3}{|c|}{ Patients } \\
\hline & $d f$ & $\mathrm{~F}$ & P-value & $d f$ & $\mathrm{~F}$ & $\mathrm{P}$-value \\
\hline \multicolumn{7}{|c|}{ Administration effect } \\
\hline $\begin{array}{c}\text { Proximal skin } \\
\text { temperature }\end{array}$ & 1 & 0.03 & 0.88 & 1 & 6.41 & $0.04^{*}$ \\
\hline $\begin{array}{l}\text { Distal skin } \\
\text { temperature }\end{array}$ & 1 & 0.48 & 0.51 & 1 & 2.11 & 0.19 \\
\hline DPG & 1 & 1.41 & 0.27 & 1 & 0.46 & 0.52 \\
\hline $\begin{array}{l}\text { Core body } \\
\text { temperature }\end{array}$ & 1 & 0.01 & 0.91 & 1 & 2.07 & 0.19 \\
\hline \multicolumn{7}{|c|}{ Administration by time of day effect } \\
\hline $\begin{array}{c}\text { Proximal skin } \\
\text { temperature }\end{array}$ & 5.06 & 0.61 & 0.70 & 4.80 & 2.22 & 0.08 \\
\hline $\begin{array}{l}\text { Distal skin } \\
\text { temperature }\end{array}$ & 6.48 & 0.37 & 0.91 & 11.05 & 1.36 & 0.21 \\
\hline DPG & 6.74 & 0.33 & 0.93 & 9.44 & 1.56 & 0.14 \\
\hline $\begin{array}{l}\text { Core body } \\
\text { temperature }\end{array}$ & 5.74 & 1.65 & 0.16 & 7.58 & 1.56 & 0.16 \\
\hline
\end{tabular}

with controls. In patients, SXB increased nocturnal SWS, normalised proximal skin temperature, and strengthened the relationship between changes in skin temperature and subsequent daytime sleep onset.

\section{An altered thermoregulatory profile in narcolepsy}

In the present study, core body temperature and proximal skin temperature were lower in narcolepsy, mainly caused by significant differences during daytime. No significant differences in distal skin temperature were found, although the nocturnal time course of distal skin temperature significantly differed between patients and controls.

The finding of a decreased daytime proximal skin temperature in patients with narcolepsy compared with controls was previously demonstrated as well (Fronczek et al., 2006). In contrast to our current findings, this previous work also reported a higher distal skin temperature. The combination of the increased distal skin temperature and the decreased proximal skin temperature in that study resulted in a higher DPG. Comparison of the present study with the previous one indicates that the absence of a higher distal skin temperature in patients, and subsequently the absence of a higher DPG, is mainly due to a higher distal skin temperature in controls in the current study. Because a higher distal skin temperature can be a direct consequence of a supine position (Tikuisis and Ducharme, 1996), maintaining this position throughout our study can be the explanation of the higher distal skin temperature found in controls. Consequently, these results indicate that patients with narcolepsy are likely to attain, even in an upright or sitting position, the high distal skin temper- ature that healthy controls reach only when remaining in a supine position.

We found a lower core body temperature during the day in patients. In healthy controls, a lower core body temperature is associated with sleep, and would theoretically result in a lower ability to maintain wakefulness. In the past, core body temperature has been more extensively studied than skin temperature. Unfortunately, previous studies in narcolepsy are not conclusive at this point; results vary from an elevated core body temperature to a lowered core body temperature (Dantz et al., 1994; Fronczek et al., 2008b; Grimaldi et al., 2010; Mosko et al., 1983; Pollak and Wagner, 1994). Manipulation studies demonstrate a minimal effect of manipulation of core body temperature on sleep propensity, (Fronczek et al., 2008b; Raymann et al., 2005; van Someren, 2006); however, a high core body temperature is associated with higher vigilance (Fronczek et al., 2008b; Raymann et al., 2007).

\section{SXB normalises temperature profiles in narcolepsy}

In patients, SXB administration significantly increased daytime proximal skin temperature, reaching levels comparable with healthy controls. In controls, proximal skin warming resulted in decreased sleep-onset latency (Raymann et al., 2005, 2008). In narcolepsy, however, despite the previously described lowered daytime proximal skin temperature, no beneficial effect of daytime proximal skin warming was found (Fronczek et al., 2008b). These findings do not point to changes in temperature as the primary mechanism through which SXB reduces the amount of daytime sleep attacks (Lammers et al., 1993). Warming up the skin by direct manipulation may represent a different physiological mechanism compared with the intrinsic skin warming resulting from SXB administration.

However, two other mechanisms might explain the positive effects of these temperature changes as a consequence of SXB administration. First, SXB induces more consolidation of sleep, i.e. probably lowers the known increased sleep stage shift index (van der Meijden et al., 2014). Second, although for a short time, in high dosages SXB is reported to increase the sympathetic response (Hicks et al., 2004; Reed and Clegg, 2006), while a chronically decreased sympathetic distal vasoconstrictor tone was hypothesised to be causal to the previously found increased DPG and the subsequently increased sleep propensity (Fronczek et al., 2006).

In healthy subjects, sleep onset is preceded by a decline in core body temperature and an increase in distal skin temperature (Raymann et al., 2007). In healthy controls, an increased DPG is associated with a lower vigilance (Ramautar et al., 2013; Romeijn and van Someren, 2011) and an accelerated sleep onset (Krauchi et al., 1999, 2000). In narcolepsy, a shorter sleep-onset latency was found to be associated with an increase of proximal and distal skin temperatures and, to a lesser extent, an increase of the DPG (Fronczek et al., 2006). However, none of these studies 


\begin{tabular}{|c|c|c|c|c|c|c|}
\hline & \multicolumn{3}{|l|}{ Baseline } & \multicolumn{3}{|c|}{ SXB administration } \\
\hline & Estimate & $S E$ & P-value & Estimate & $S E$ & P-value \\
\hline \multicolumn{7}{|l|}{ Proximal skin temperature } \\
\hline At $30 \mathrm{~s}$ prior to sleep onset & 0.0 & 0.1 & 0.588 & 0.1 & 0.1 & $0.013^{*}$ \\
\hline Change 5 min prior to sleep onset & 3.7 & 1.4 & $0.006^{\star \star}$ & 0.9 & 1.4 & 0.518 \\
\hline Change 15 min prior to sleep onset & -1.5 & 0.8 & 0.064 & 0.1 & 0.8 & 0.948 \\
\hline \multicolumn{7}{|l|}{ Distal skin temperature } \\
\hline At $30 \mathrm{~s}$ prior to sleep onset & 0.1 & 0.1 & 0.479 & 0.4 & 0.1 & $0.002^{* *}$ \\
\hline Change 5 min prior to sleep onset & -0.3 & 0.6 & 0.571 & 1.2 & 0.7 & 0.110 \\
\hline Change $15 \mathrm{~min}$ prior to sleep onset & 0.8 & 0.4 & $0.029^{*}$ & 1.4 & 0.4 & $0.002^{* *}$ \\
\hline \multicolumn{7}{|l|}{ DPG } \\
\hline At $30 \mathrm{~s}$ prior to sleep onset & 0.1 & 0.1 & 0.578 & 0.3 & 0.1 & $0.020^{*}$ \\
\hline Change 5 min prior to sleep onset & -0.2 & 0.6 & 0.744 & 1.0 & 0.7 & 0.143 \\
\hline Change $15 \mathrm{~min}$ prior to sleep onset & 0.8 & 0.4 & $0.027^{*}$ & 1.2 & 0.4 & $0.005^{\star *}$ \\
\hline \multicolumn{7}{|l|}{ Core body temperature } \\
\hline At $30 \mathrm{~s}$ prior to sleep onset & 0.0 & 0.0 & 0.405 & 0.0 & 0.0 & 0.925 \\
\hline Change 5 min prior to sleep onset & -0.1 & 2.2 & 0.957 & -0.7 & 2.6 & 0.787 \\
\hline Change $15 \mathrm{~min}$ prior to sleep onset & 0.6 & 1.3 & 0.666 & -1.6 & 1.6 & 0.326 \\
\hline \multicolumn{7}{|c|}{$\begin{array}{l}\text { Results of linear mixed effects analysis for patients at baseline and patients during SXB administration (nights were excluded), indicating } \\
\text { effects of temperature fluctuations as regressor for fluctuations in nap probability. Analysis was performed for proximal skin temperature, } \\
\text { distal skin temperature, distal-proximal temperature gradient (DPG) or core body temperature at the moments: difference between the } \\
\text { temperature during the } 30 \text {-s epoch prior to sleep onset and } 15 \text { min prior to sleep onset; difference between the temperature during the } 30 \text {-s } \\
\text { epoch prior to sleep onset and } 5 \text { min prior to sleep onset; or the absolute temperature during the } 30 \text {-s epoch prior to sleep onset. } \\
\text { SXB, sodium oxybate. } \\
* P<0.05 ; * * P<0.01 \text {. }\end{array}$} \\
\hline
\end{tabular}

concerned spontaneous daytime napping in patients with narcolepsy. Analysis of spontaneous naps in the (semi) supine position in the present study revealed a predictive value for proximal skin temperature, distal skin temperature and DPG in patients with narcolepsy at baseline and during SXB administration. This relationship between an increase of skin temperature and subsequent sleep onset is known to exist in controls, exists in patients with narcolepsy as well, and is even more clear after SXB administration.

\section{Does altered thermoregulation play a role in the effects of SXB on sleep?}

An increase in nocturnal SWS, previously reported to be one of the principal effects of SXB on sleep (Broughton and Mamelak, 1980; Lammers et al., 1993; Lapierre et al., 1990; Scrima et al., 1990), was confirmed in this study. If this is mediated by an altered temperature regulation is questionable, as there were no nocturnal temperature effects seen during SXB intake in this study. The relatively high percentage of wake during the night in controls is probably due to the laboratory settings.

\section{Study limitations}

Because body position directly affects skin temperature, the major limitation of this study is the setting in which patients were in the (semi)supine position for $24 \mathrm{~h}$. This body position differs from the situation in normal daily life, and the setting in previous studies. Moreover, the clinical effects of SXB on nocturnal sleep can already be experienced with the dose we have used in the first night of its use, but it usually takes several weeks and a higher dose to obtain optimal clinical improvement, and significant improvement of cataplexy and EDS. Subsequently, it is presumable that there are some important long-term effects, particularly during daytime that may have been missed in this study. Furthermore, the present study included a relatively small number of patients (of whom two were drug-naive and the others discontinued treatment) and only male subjects, while men and women are equally affected with narcolepsy. This might have lead to an underestimation of the actual effects of SXB and limits the generalisation of the results.

\section{CONCLUSION}

In conclusion, during a constant routine protocol a decreased daytime core body and proximal skin temperature were observed in patients with narcolepsy compared with controls. Administration of SXB improved the sleep-wake pattern, and partially normalised the temperature profiles in patients with narcolepsy. Furthermore, SXB strengthened the relationship between skin temperature and subsequent sleep onset (that is known to exist in controls) in patients. To further explore the role of SXB in temperature regulation and sleep in narcolepsy, studies with patients and controls of both sexes have to be performed in normal daily life. 


\section{ACKNOWLEDGEMENTS}

This study was supported by UCB Pharma. The authors are greatly indebted to the volunteers who participated in this study. In addition, the authors would like to thank E. J. M. Ladan-Eygenraam, J. van Vliet-de Regt and P. J. van Someren for technical assistance during the study.

\section{AUTHOR CONTRIBUTIONS}

AvdH: data collection, data analysis, interpretation of results and preparation of manuscript; CD: data collection, interpretation of results and preparation of manuscript; HP: study design, interpretation of results and preparation of manuscript; RR: data analysis, interpretation of results and preparation of manuscript; SO: study design, interpretation of results and preparation of manuscript; GJL: study design, interpretation of results and preparation of manuscript; EvS: study design, interpretation of results and preparation of manuscript; RF: study design, interpretation of results and preparation of manuscript.

\section{CONFLICT OF INTEREST}

This study was supported by UCB Pharma. Dr Lammers has served as a paid member of the UCB advisory board, and received lecture fees and conference travel support from UCB Pharma. Dr Lammers also did consultancy for UCB Pharma, and provided expert testimony for UCB Pharma, Jazz Pharmaceuticals, and Bioprojet. Dr Overeem has served as a paid member of the UCB advisory board, and received lecture fees from UCB Pharma, Novartis, and Boehringer Ingelheim. He also received conference travel support from UCB Pharma. R. Fronczek, A. van der Heide and C. E. H. M. Donjacour received conference travel support from UCB Pharma. The other authors report no financial conflict of interest. For this study we used SXB (Xyrem) from UCB Pharma.

\section{REFERENCES}

American Academy of Sleep Medicine. International Classification of Sleep Disorders - Third Edition (ICSD-3). American Academy of Sleep Medicine, Darien, IL, 2014.

Billiard, M., Bassetti, C., Dauvilliers, Y. et al. EFNS guidelines on management of narcolepsy. Eur. J. Neurol., 2006, 13: 1035-1048.

Broughton, R. and Mamelak, M. Effects of nocturnal gammahydroxybutyrate on sleep/waking patterns in narcolepsy-cataplexy. Can. J. Neurol. Sci., 1980, 7: 23-31.

Byrne, C. and Lim, C. L. The ingestible telemetric body core temperature sensor: a review of validity and exercise applications. Br. J. Sports Med., 2007, 41: 126-133.

Chin, R. L., Sporer, K. A., Cullison, B., Dyer, J. E. and Wu, T. D. Clinical course of gamma-hydroxybutyrate overdose. Ann. Emerg. Med., 1998, 31: 716-722.

Dantz, B., Edgar, D. M. and Dement, W. C. Circadian rhythms in narcolepsy: studies on a 90 minute day. Electroencephalogr. Clin. Neurophysiol., 1994, 90: 24-35.

Dauvilliers, Y., Arnulf, I. and Mignot, E. Narcolepsy with cataplexy. Lancet, 2007, 369: 499-511.
Donjacour, C. E., Aziz, N. A., Frolich, M. et al. Sodium oxybate increases prolactin secretion in narcolepsy patients and healthy controls. Eur. J. Endocrinol., 2011a, 164: 363-370.

Donjacour, C. E., Aziz, N. A., Roelfsema, F. et al. Effect of sodium oxybate on growth hormone secretion in narcolepsy patients and healthy controls. Am. J. Physiol. Endocrinol. Metab., 2011b, 300: E1069-E1075.

Donjacour, C. E. H. M., Kalsbeek, A., Overeem, S. et al. Altered circadian rhythm of melatonin concentrations in hypocretin-deficient men. Chronobiol. Int., 2012, 29: 356-362.

Fronczek, R., Overeem, S., Lammers, G. J., van Dijk, J. G. and Van Someren, E. J. W. Altered skin-temperature regulation in narcolepsy relates to sleep propensity. Sleep, 2006, 29: 1444 1449.

Fronczek, R., Raymann, R. J. E. M., Overeem, S. et al. Manipulation of skin temperature improves nocturnal sleep in narcolepsy. $J$. Neurol. Neurosurg. Psychiatry, 2008a, 79: 1354-1357.

Fronczek, R., Raymann, R. J. E. M., Romeijn, N. et al. Manipulation of core body and skin temperature improves vigilance and maintenance of wakefulness in narcolepsy. Sleep, 2008b, 31: 233-240.

Grimaldi, D., Agati, P., Pierangeli, G. et al. Hypocretin deficiency in narcolepsy with cataplexy is associated with a normal body core temperature modulation. Chronobiol. Int., 2010, 27: 1596-1608.

Hicks, A. R., Kapusta, D. R. and Varner, K. J. Mechanisms underlying the sympathomimetic cardiovascular responses elicited by gamma-hydroxybutyrate. J. Cardiovasc. Pharmacol., 2004, 44: 631-638.

Iber, C., Ancoli-Israel, S., Chesson, A. L. Jr and Quan, S. F. for the American Academy of Sleep Medicine. The AASM Manual for the Scoring of Sleep and Associated Events: Rules, Terminology and Technical Specifications. American Academy of Sleep Medicine, Westchester, IL, 2007.

Kaufman, E. E., Porrino, L. J. and Nelson, T. Pyretic action of low doses of gamma-hydroxybutyrate in rats. Biochem. Pharmacol., 1990, 40: 2637-2640.

Krauchi, K., Cajochen, C., Werth, E. and Wirz-Justice, A. Warm feet promote the rapid onset of sleep. Nature, 1999, 401: 36-37.

Krauchi, K., Cajochen, C., Werth, E. and Wirz-Justice, A. Functional link between distal vasodilatation and sleep-onset latency? Am. J. Physiol. Regul. Integr. Comp. Physiol., 2000, 278: R741-R748.

Krul, J. and Girbes, A. R. Gamma-hydroxybutyrate: experience of 9 years of gamma-hydroxybutyrate (GHB)-related incidents during rave parties in The Netherlands. Clin. Toxicol. (Phila.), 2011, 49: 311-315.

Lammers, G. J., Arends, J., Declerck, A. C., Ferrari, M. D., Schouwink, G. and Troost, J. Gammahydroxybutyrate and narcolepsy: a double-blind placebo-controlled study. Sleep, 1993, 16: 216-220.

Lapierre, O., Montplaisir, J., Lamarre, M. and Bedard, M. A. The effect of gamma-hydroxybutyrate on nocturnal and diurnal sleep of normal subjects: further considerations on REM sleep-triggering mechanisms. Sleep, 1990, 13: 24-30.

van Marken Lichtenbelt, W. D., Daanen, H. A., Wouters, L. et al. Evaluation of wireless determination of skin temperature using iButtons. Physiol. Behav., 2006, 88: 489-497.

Mayer, G., Hellmann, F., Leonhard, E. and Meier-Ewert, K. Circadian temperature and activity rhythms in unmedicated narcoleptic patients. Pharmacol. Biochem. Behav., 1997, 58: 395-402.

van der Meijden, W. P., Fronczek, R., Reijntjes, R. H. A. M. et al. Time- and state-dependent analysis of autonomic control in narcolepsy: higher heart rate with normal heart rate variability independent of sleep fragmentation. J. Sleep Res., 2015, 24: 206214.

Mosko, S. S., Holowach, J. B. and Sassin, J. F. The 24-hour rhythm core temperature in narcolepsy. Sleep, 1983, 6: 137-146. 
Nishino, S., Ripley, B., Overeem, S., Lammers, G. J. and Mignot, E. Hypocretin (orexin) deficiency in human narcolepsy. Lancet, 2000, 355: 39-40.

Pollak, C. P. and Wagner, D. R. Core body temperature in narcoleptic and normal subjects living in temporal isolation. Pharmacol. Biochem. Behav., 1994, 47: 65-71.

Ramautar, J. R., Romeijn, N., Gómez-Herrero, G., Piantoni, G. and Van Someren, E. J. W. Coupling of infraslow fluctuations in autonomic and central vigilance markers: skin temperature, EEG beta power and ERP P300 latency. Int. J. Psychophysiol., 2013, 89: 158-164.

Raymann, R. J., Swaab, D. F. and van Someren, E. J. Cutaneous warming promotes sleep onset. Am. J. Physiol. Regul. Integr. Comp. Physiol., 2005, 288: R1589-R1597.

Raymann, R. J., Swaab, D. F. and van Someren, E. J. Skin temperature and sleep-onset latency: changes with age and insomnia. Physiol. Behav., 2007, 90: 257-266.

Raymann, R. J., Swaab, D. F. and van Someren, E. J. Skin deep: enhanced sleep depth by cutaneous temperature manipulation. Brain, 2008, 131: 500-513.

Reed, M. J. and Clegg, G. R. Paroxysmal sympathetic surge associated with gamma hydroxybutyrate. Eur. J. Emerg. Med., 2006, 13: 41-42.
Romeijn, N. and van Someren, E. J. Correlated fluctuations of daytime skin temperature and vigilance. J. Biol. Rhythms, 2011, 26: 68-77.

Romeijn, N., Raymann, R. J., Most, E. et al. Sleep, vigilance, and thermosensitivity. Pflugers Arch., 2012, 463: 169-176.

Scrima, L., Hartman, P. G., Johnson, F. H. J., Thomas, E. E. and Hiller, F. C. The effects of gamma-hydroxybutyrate on the sleep of narcolepsy patients: a double-blind study. Sleep, 1990, 13: 479490.

van Someren, E. J. More than a marker: interaction between the circadian regulation of temperature and sleep, age-related changes, and treatment possibilities. Chronobiol. Int., 2000, 17: 313-354.

van Someren, E. J. Mechanisms and functions of coupling between sleep and temperature rhythms. Prog. Brain Res., 2006, 153: 309324.

Tikuisis, P. and Ducharme, M. B. The effect of postural changes on body temperatures and heat balance. Eur. J. Appl. Physiol. Occup. Physiol., 1996, 72: 451-459. 